\title{
Quality by design of yogurt product using taguchi multi responses method
}

\author{
Ali Parkhan ${ }^{\mathrm{a}}$, Muhammad Ridwan Andi Purnomo ${ }^{\mathrm{a}^{*}}$ \\ aDepartment of Industrial Engineering, Universitas Islam Indonesia, Yogyakarta, Indonesia.
}

${ }^{*}$ Corresponding Author: ridwan_ie@uii.ac.id

\section{ARTICLE INFO}

\section{Keywords}

Yogurt quality improvement; Design of experiments;

Taguchi method; Multi responses signal to noise.

\section{Article history}

Received:

01 July, 2020

Revised:

15 July, 2020

Accepted:

05 August, 2020

Available online:

21 August, 2020

\begin{abstract}
Quality of yogurt could be determined based on its flavor and texture which could be tested using an organoleptic test. The quality objective of the yogurt flavor and texture is larger the better. As one of the intermediate products that could be processed to become various other products, the demand for yogurt is continuously increasing in Indonesia. Along with the increase in demand, the demand for quality of yogurt has also increased. In this study, experiments have been conducted to improve the quality of a yogurt product. The experiments were designed based on the Taguchi method with Multi Responses Signal to Noise (MRSN) that involves 7 factors consists of 6 controllable factors and 1 uncontrollable factor. Every factor has 2 levels of an experiment. The 6 controllable factors are heating temperature, heating duration, number of yogurt seeds, incubation temperature, incubation duration, number of sugars, while the uncontrollable factor is the weather condition. Result of the experiments showed when weight for flavor and texture is 0.437 and 0.563 respectively, the levels of the optimum factors are $95^{\circ} \mathrm{C}$ for milk heating temperature, 20 minutes for the duration of milk heating process, $75 \mathrm{ml}$ of yogurt seeds, $45^{\circ} \mathrm{C}$ of the temperature of incubation, 6 hours of incubation duration and 12.5 grams of sugar weight. Based on the organoleptic test that conducted by a group of experienced testers, the new optimum factors combination could improve the yogurt's quality in term of flavor and quality up to $16.24 \%$ and $11.37 \%$ respectively. It could be concluded that the proposed method could improve the quality of yogurt-based on preferences from the experienced testers that have been expressed by the weight of every quality response.
\end{abstract}

\section{Introduction}

Milk is nutrition that has high nutritional content, and because of its multifunctional properties, its dairy proteins could be used to enhance the texture of some food products (Lesme, Rannou, Famelart, Bouhallab, \& Prost, 2020). Besides, milk could be consumed by people directly, therefore, its demand remains high from time to time. Milk has a perfect composition and it makes milk become a very good medium for the growth of organisms. Milk consumption usually is indirect; the milk will be stored before consumption. When being stored, there will be a lot of microorganisms grow, and they will contaminate the milk. It is very important to control the milk condition during the storing period to avoid that problem, and basically, that problem could be addressed by processing and preserving the milk. One of the techniques is fermenting the milk into yogurt using lactic acid bacteria at a controlled temperature. In the fermentation process, temperature control is needed to enable bacteria growth optimally. The bacteria would change 
natural milk sugar and release lactic acid as waste. High acidity causes denaturation of milk protein and it produces a dense texture on yogurt. High acidity $(\mathrm{pH}=4-5)$ also makes proliferation of potential pathogenic bacteria.

Besides fermentation temperature, another controllable factor that affects yogurt quality is heating duration. In the yogurt fermentation process, the number of bacteria and yogurt seeds growth must be controlled. When heating duration is short, the number of bacteria that still alive would be much and they could dominate the yogurt seeds. However, the long heating duration would evaporate a lot of water and affect the milk density and it could resist the growth of yogurt seeds.

The controllable factor that affects yogurt quality is the number of yogurt seeds. When the number of yogurt seeds is few then it would affect the speed of the lactose disperse and it affects the yogurt flavor. However, when the number of yogurt seeds is much, it will cause excessive production of lactic acid and the yogurt would be very acidic. After the yogurt is produced, then it must be incubated for a while. The incubation time is also affecting yogurt quality. Therefore, incubation time must be optimized through some experiments. When incubation time is short, it affects to yogurt density and flavor. However, when the incubation time is long then it will cause excessive production of lactic acid as happened when the number of yogurt seeds is much. The last controllable factor that affects yogurt quality is the number of sugars. Little sugar would affect the flavor of yogurt while much sugar would affect the number of lactic acid bacteria. Based on a preliminary experiment, besides the 6 controllable factors, there is an uncontrollable factor that could not be avoided that is the weather.

The objective of this study is to design the quality of yogurt products by experiment. In this study, 2 quality responses of the yogurt were considered; those were flavor and texture; with quality, the objective is larger the better. There are 7 input factors considered and experiments to get an optimum setting of the input factors were designed using the Taguchi Multi Responses Signal to Noise (T-MRSN) method. The organoleptic test was used to justify both quality responses, and to reduce subjectivity judgment, a group of experienced testers was involved to carry out the test and determine the weight of every quality responses. The following sections describe the research position compared to previous studies, research methods, and data processing in designing the quality of the yogurt product.

\section{Literature Review}

Yogurt has become an interesting product to be processed due to its potentiality to be processed further become other products. However, there are several issues, which negatively affect yogurt quality and customer acceptance (Wang, Kristo, \& LaPointe, 2020). There are several previous studies regarding yogurt quality improvement, and one of them is an improvement on the yogurt production process that is in the fermentation process. Usually, two factors in the fermentation process were controlled, which are fermentation temperature (Tribs, Falcade, Carvalho, de Castro Leite Junior, \& de Oliveira, 2020) and fermentation duration. Since yogurt quality is determined based on its ingredient and the making process, then most of the previous studies were implemented experiments to get initial data and statistical analysis to identify the process environment pattern (Lordan, Vidal, Pham, Tsoupras, Thomas, \& Zabetakis, 2020; Anuyahong, Chusak, \& Adisakwattana, 2020). Therefore, in this study, a Taguchi method with multi responses objective would be used as the basis for designing the experiments.

The Taguchi method has been widely applied to improve product quality by inserting quality in the production process. Several previous studies have implemented Taguchi method to optimize process parameters, such as welding process (Shunmugasundaram, Praveen Kumar, Ponraj Sankar, \& Sivasankar, 2020), wire electrical discharge machining (WEDM) (Fakkir Mohamed \& 
Lenin, 2020), surface and turning machining process (Khare, Phull, \& Agarwal, 2020; Ramesh, Singh, Gnanasekaran, \& Kumar, 2020), concrete block production process (Ikeagwuani, Nwonu, Ugwu, \& Agu, 2020) and laser bending of a metal (Behera, Sahu, \& Patel, 2020). Taguchi method offers a robust and effective experiment design by considering uncontrollable factors that always exist in every production process. This method is more effective compared to the traditional experiment method that uses a full combination of factors. Therefore, the Taguchi method would be implemented in this study to design the quality of yogurt products with multi quality responses. To the best of the author's knowledge, this study has not been investigated by previous researchers and it would be the novelty of this study.

\section{Research Methodology}

Two phases have been carried out; those are experiment design and experiment operational. In the first phase, two designs have been determined, which are quality response and factors that affect the quality response. The used quality responses in this study are the flavor and texture of the yogurt. The standard for the flavor is slightly sour and not bitter while the standard for texture is soft, condensed, and porridge-like. Since the standard is vague, then a group of experienced testers was involved to justify the standard. An organoleptic test was also used as the method for the standard justification. The quality objective for the quality response is larger the better (LTB). The second design is factor selection that affects the quality response. This design was carried out based on preliminary experiments, and there are 6 controllable factors selected, that are heating temperature, heating duration, number of yogurt seeds, incubation temperature, incubation time, and number of used sugars. Besides the controllable factor, there is an uncontrollable factor in the yogurt making process that is the weather. There are 2 experiment levels for every factor that would be considered when conducting the experiments.

The second phase of this study is experiment operational. In this study, inner array $L_{8} 2^{7}$ and outer array $L_{3} 2^{4}$ was used. Experiment results are considered as a sample from a population that is the full combination of all of the factors. Therefore, it is very important to ensure that the sample has been drawn from a normally distributed population. Besides that, the sample also needs to be tested for its homogeneity to ensure that the sample has the same distribution as the other sample from the same population. In multi factors and multi-response experiments, the accuracy of the experiment is affected by the contribution of the factors to the responses. An experiment would be accurate when the factors have a significant contribution to the response. One of the methods to identify the contribution of the factors to the responses is the ANOVA test.

The next step is the determination of Signal to Noise (SNR) ratio that compares the level of the desired signal to the level of background noise. Following formula was used to calculate the SNR to every quality response (Belavendram, 1995):

$$
\text { Larger-the-Better (LTB): } S / N_{-} L T B=-\log \left[1 / n \sum_{i=n}^{n} 1 / y i^{2}\right]
$$

Where:

$n=$ number of trials

$y_{i}=$ response $i$ value

To determine the best level factors combination, the effect of every factor to the quality response must be determined. The following formula was used to calculate the effect of every factor to the quality response (Belavendram, 1995), while Table 7 and Table 8 shows the result. 


$$
\text { Factor effect }=\frac{1}{a}\left(\sum \eta_{0}\right)
$$

Where:

$a=$ number of factor level in the orthogonal matrix

$\eta=$ SNR value

$0=$ number of experiments that has the same level

\section{Results and Discussion}

In this study, the level setting was determined based on preliminary experiments. The level setting that affects the yogurt product significantly was selected, and it is shown in Table 1.

Table 1. Factors and Experiment Level Factor

\begin{tabular}{ccccc} 
FACTOR TYPE & FACTOR & CODE & LEVEL 1 & LEVEL 2 \\
\hline CONTROLLABLE & Heating temperature & A & $70^{\circ} \mathrm{C}$ & $95^{\circ} \mathrm{C}$ \\
& Heating duration & B & 20 minutes & 30 minutes \\
& Number of yogurt seeds & C & $7.5 \mathrm{ml}$ & $12.5 \mathrm{ml}$ \\
& Incubation temperature & D & $40^{\circ} \mathrm{C}$ & $45^{\circ} \mathrm{C}$ \\
& Incubation duration & $\mathrm{E}$ & 4 hours & 6 hours \\
& Number of sugars & F & 12.5 gram & 17.5 gram \\
UNCONTROLLABLE & Weather & H & Rainy & Cloudy
\end{tabular}

The experiment results using inner array $L_{8} 2^{7}$ and outer array $L_{3} 2^{4}$ for every quality response are shown in Table 2 and Table 3 below.

Table 2. Experiment Result for Quality Response: Flavor

\begin{tabular}{|c|c|c|c|c|c|c|c|c|c|c|c|c|c|c|c|}
\hline \multirow{4}{*}{ TRIAL } & \multicolumn{7}{|c|}{ CONTROLLABLE FACTORS } & \multicolumn{8}{|c|}{ UNCONTROLLABLE FACTOR } \\
\hline & A & B & C & D & $\mathbf{E}$ & $\mathbf{F}$ & ER & \multicolumn{4}{|c|}{ EXPERIMENT RESULT } & \multicolumn{4}{|c|}{ EXPERIMENT RESULT } \\
\hline & \multicolumn{7}{|c|}{ COLUMN NO. } & \multicolumn{4}{|c|}{ REPLICATION } & \multicolumn{4}{|c|}{ REPLICATION } \\
\hline & 1 & 2 & 3 & 4 & 5 & 6 & 7 & 1 & 2 & 3 & 4 & 1 & 2 & 3 & 4 \\
\hline 1 & 1 & 1 & 1 & 1 & 1 & 1 & 1 & 1.286 & 1.429 & 1.571 & 1.286 & 1.571 & 1.857 & 2.000 & 1.571 \\
\hline 2 & 1 & 1 & 1 & 2 & 2 & 2 & 2 & 2.429 & 2.429 & 2.000 & 1.571 & 2.286 & 2.000 & 2.857 & 2.857 \\
\hline 3 & 1 & 2 & 2 & 1 & 1 & 2 & 2 & 1.571 & 2.000 & 1.571 & 1.571 & 1.571 & 1.571 & 1.571 & 1.571 \\
\hline 4 & 1 & 2 & 2 & 2 & 2 & 1 & 1 & 2.429 & 1.857 & 2.714 & 2.714 & 2.714 & 2.857 & 2.286 & 2.429 \\
\hline 5 & 2 & 1 & 2 & 1 & 2 & 1 & 2 & 2.714 & 2.857 & 2.429 & 2.429 & 2.286 & 2.429 & 2.286 & 2.000 \\
\hline 6 & 2 & 1 & 2 & 2 & 1 & 2 & 1 & 2.000 & 2.714 & 1.857 & 1.571 & 1.286 & 1.571 & 1.571 & 1.857 \\
\hline 7 & 2 & 2 & 1 & 1 & 2 & 2 & 1 & 1.857 & 2.000 & 1.286 & 1.857 & 1.429 & 1.286 & 2.000 & 1.571 \\
\hline 8 & 2 & 2 & 1 & 2 & 1 & 1 & 2 & 2.714 & 2.286 & 2.000 & 2.000 & 2.000 & 1.571 & 2.286 & 2.429 \\
\hline
\end{tabular}


Table 3. Experiment Result for Quality Response: Texture

\begin{tabular}{|c|c|c|c|c|c|c|c|c|c|c|c|c|c|c|c|}
\hline \multirow{4}{*}{ TRIAL } & \multicolumn{7}{|c|}{ CONTROLLABLE FACTORS } & \multicolumn{8}{|c|}{ UNCONTROLLABLE FACTOR } \\
\hline & A & B & C & D & E & $\mathbf{F}$ & ER & \multicolumn{4}{|c|}{ EXPERIMENT RESULT } & \multicolumn{4}{|c|}{ EXPERIMENT RESULT } \\
\hline & \multicolumn{7}{|c|}{ COLUMN NO. } & \multicolumn{4}{|c|}{ REPLICATION } & \multicolumn{4}{|c|}{ REPLICATION } \\
\hline & 1 & 2 & 3 & 4 & 5 & 6 & 7 & 1 & 2 & 3 & 4 & 1 & 2 & 3 & 4 \\
\hline 1 & 1 & 1 & 1 & 1 & 1 & 1 & 1 & 1.000 & 1.143 & 1.000 & 1.143 & 1.143 & 1.000 & 1.143 & 1.714 \\
\hline 2 & 1 & 1 & 1 & 2 & 2 & 2 & 2 & 2.429 & 1.857 & 1.429 & 2.429 & 2.714 & 2.429 & 2.429 & 2.429 \\
\hline 3 & 1 & 2 & 2 & 1 & 1 & 2 & 2 & 1.143 & 1.429 & 1.143 & 1.714 & 1.714 & 1.571 & 1.143 & 1.429 \\
\hline 4 & 1 & 2 & 2 & 2 & 2 & 1 & 1 & 2.429 & 1.429 & 2.857 & 2.714 & 2.714 & 1.429 & 1.857 & 1.857 \\
\hline 5 & 2 & 1 & 2 & 1 & 2 & 1 & 2 & 1.714 & 2.429 & 2.000 & 2.143 & 1.571 & 1.857 & 2.000 & 2.143 \\
\hline 6 & 2 & 1 & 2 & 2 & 1 & 2 & 1 & 2.429 & 2.714 & 2.143 & 2.429 & 2.000 & 2.429 & 2.143 & 2.429 \\
\hline 7 & 2 & 2 & 1 & 1 & 2 & 2 & 1 & 1.714 & 2.857 & 2.428 & 2.857 & 2.000 & 2.143 & 2.143 & 2.429 \\
\hline 8 & 2 & 2 & 1 & 2 & 1 & 1 & 2 & 2.429 & 2.714 & 2.000 & 2.429 & 2.143 & 2.143 & 2.000 & 2.857 \\
\hline
\end{tabular}

The normality and homogeneity test for the experiment result with $X$ critical value is 7.815 and 3.842 respectively shows that the experiment result is normal and homogeny. ANOVA test with $F$ critical value is 4.013 shows that factor $D, E$, and $F$ has a significant contribution to the flavor, while factor $A, D$, and $E$ has a significant contribution to the texture. It means that all of the controllable factors are need to be considered to control the quality of flavor and texture of the yogurt. Table 4 below shows the SNR value for every experiment trial while Table 5 and Table 6 show the effect of every controllable factor on the quality response.

Table 4. SNR Value for Every Experiment Trial

\begin{tabular}{cccccccccc} 
& \multicolumn{1}{c}{ TRIAL } & \multicolumn{1}{c}{ CONTROLLABLE FACTORS } & \multicolumn{3}{c}{ SNR } \\
\cline { 2 - 9 } & A & B & C & D & E & F & ER & FLAVOR & TEXTURE \\
\hline 1 & 1 & 1 & 1 & 1 & 1 & 1 & 1 & 3.647 & 0.968 \\
2 & 1 & 1 & 1 & 2 & 2 & 2 & 2 & 6.776 & 6.569 \\
3 & 1 & 2 & 2 & 1 & 1 & 2 & 2 & 4.137 & 2.634 \\
4 & 1 & 2 & 2 & 2 & 2 & 1 & 1 & 7.727 & 5.762 \\
5 & 2 & 1 & 2 & 1 & 2 & 1 & 2 & 7.571 & 5.726 \\
6 & 2 & 1 & 2 & 2 & 1 & 2 & 1 & 4.581 & 7.272 \\
7 & 2 & 2 & 1 & 1 & 2 & 2 & 1 & 4.001 & 6.963 \\
8 & 2 & 2 & 1 & 2 & 1 & 1 & 2 & 6.375 & 7.176
\end{tabular}

Table 5. Effect of Every Controllable Factor to The Quality Response: Flavor

\begin{tabular}{lcccccc}
\multirow{2}{*}{ LEVEL } & \multicolumn{6}{c}{ CONTROLLABLE FACTOR } \\
\cline { 2 - 7 } & $\mathbf{A}$ & $\mathbf{B}$ & $\mathbf{C}$ & $\mathbf{D}$ & $\mathbf{E}$ & $\mathbf{F}$ \\
\hline Level 1 & 5.572 & 5.644 & 5.2 & 4.839 & 4.685 & 6.33 \\
Level 2 & 5.632 & 5.56 & 6.004 & 6.365 & 6.519 & 4.874 \\
Difference & $\mathbf{0 , 0 6 0}$ & $\mathbf{0 , 0 8 4}$ & $\mathbf{0 , 8 0 4}$ & $\mathbf{1 , 5 2 6}$ & $\mathbf{1 , 8 3 4}$ & $\mathbf{1 , 4 5 6}$
\end{tabular}


Table 6. Effect of Every Controllable Factor to The Quality Response: Texture

\begin{tabular}{lcccccc}
\multirow{2}{*}{ LEVEL } & \multicolumn{7}{c}{ CONTROLLABLE FACTOR } \\
\cline { 2 - 7 } & $\mathbf{A}$ & $\mathbf{B}$ & $\mathbf{C}$ & $\mathbf{D}$ & $\mathbf{E}$ & F \\
\hline Level 1 & 3,983 & 5,134 & 5,419 & 4,073 & 4,513 & 4,908 \\
Level 2 & 6,784 & 5,634 & 5,348 & 6,695 & 6,255 & 5,860 \\
Difference & $\mathbf{2 , 8 0 1}$ & $\mathbf{0 , 5 0 0}$ & $\mathbf{0 , 0 7 1}$ & $\mathbf{2 , 6 2 2}$ & $\mathbf{1 , 7 4 2}$ & $\mathbf{0 , 9 5 2}$
\end{tabular}

From Table 5 and Table 6 , it could be determined that the best level factors combination for flavor and texture is A2 B1 C2 D2 E2 F1 and A2 B2 C1 D2 E2 F2 respectively. Since the best level factors combination for every quality response is different, then multi responses analysis is required. The factors that affect the flavor and texture simultaneously are A, D, E, and F. Therefore, analysis on $2^{4}$ combination alternatives and their slices with $A O L_{8} 2^{7}$ is required, as depicted in the following figure.

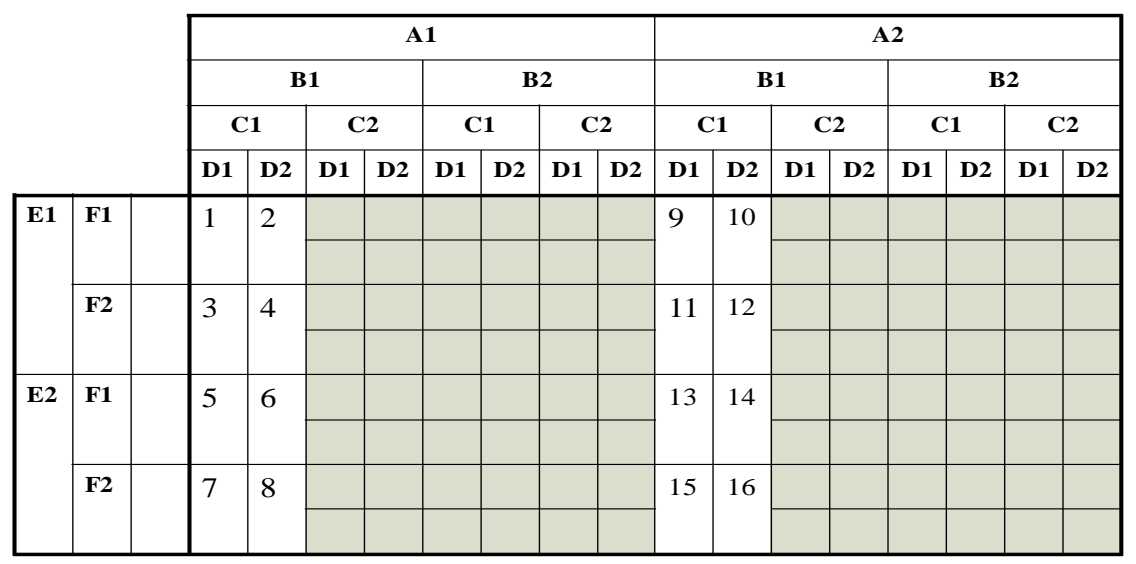

Figure 1. Matrix for Multi Responses Analysis

Besides the 16 experiments as shown in Figure 1, based on $L_{8} 2^{7}$ matrix, 6 experiments have been carried out; therefore, 22 experiments would be carried out to obtain the best level factors combination. The following table shows the level factor combinations that have been carried out.

Table 7. Level Factors Combinations for The New Experiments

\begin{tabular}{|c|c|c|c|c|c|c|c|c|c|c|c|c|c|}
\hline \multirow{2}{*}{ TRIAL } & \multicolumn{6}{|c|}{ CONTROLLABLE FACTORS } & \multirow{2}{*}{ TRIAL } & \multicolumn{6}{|c|}{ CONTROLLABLE FACTORS } \\
\hline & A & B & C & D & $E$ & $F$ & & $\mathbf{A}$ & B & C & D & $E$ & $F$ \\
\hline 1 & 1 & 1 & 1 & 1 & 1 & 1 & 12 & 2 & 1 & 1 & 2 & 1 & 2 \\
\hline 2 & 1 & 1 & 1 & 2 & 1 & 1 & 13 & 2 & 1 & 1 & 1 & 2 & 1 \\
\hline 3 & 1 & 1 & 1 & 1 & 1 & 2 & 14 & 2 & 1 & 1 & 2 & 2 & 1 \\
\hline 4 & 1 & 1 & 1 & 2 & 1 & 2 & 15 & 2 & 1 & 1 & 1 & 2 & 2 \\
\hline 5 & 1 & 1 & 1 & 1 & 2 & 1 & 16 & 2 & 1 & 1 & 2 & 2 & 2 \\
\hline 6 & 1 & 1 & 1 & 2 & 2 & 1 & 17 & 1 & 2 & 2 & 1 & 1 & 2 \\
\hline 7 & 1 & 1 & 1 & 1 & 2 & 2 & 18 & 1 & 2 & 2 & 2 & 2 & 1 \\
\hline 8 & 1 & 1 & 1 & 2 & 2 & 2 & 19 & 2 & 1 & 2 & 1 & 2 & 1 \\
\hline 9 & 2 & 1 & 1 & 1 & 1 & 1 & 20 & 2 & 1 & 2 & 2 & 1 & 2 \\
\hline 10 & 2 & 1 & 1 & 2 & 1 & 1 & 21 & 2 & 2 & 1 & 1 & 2 & 2 \\
\hline 11 & 2 & 1 & 1 & 2 & 1 & 2 & 22 & 2 & 2 & 1 & 2 & 1 & \\
\hline
\end{tabular}


Based on the result of the 22 new experiments, then double regression linear models to predict the quality responses based on the controllable factors could be obtained. Moreover, the obtained double regression linear model combined with an uncontrollable factor could be used to predict the quality responses. Table 8 and Table 9 below show the prediction results.

Table 8. Prediction of Experiments Results for Quality Response: Flavor

\begin{tabular}{|c|c|c|c|c|c|c|c|c|c|}
\hline \multirow{3}{*}{ TRIAL } & \multicolumn{8}{|c|}{ UNCONTROLLABLE FACTOR } & \multirow{3}{*}{$\begin{array}{c}\text { RESULT } \\
\text { (AVERAGE) }\end{array}$} \\
\hline & \multicolumn{4}{|c|}{ REPLICATIONS } & \multicolumn{4}{|c|}{ REPLICATIONS } & \\
\hline & 1 & 2 & 3 & 4 & 1 & 2 & 3 & 4 & \\
\hline 1 & 1.518 & 1.626 & 1.643 & 1.304 & 1.714 & 1.857 & 2.143 & 1.750 & 1.694 \\
\hline 2 & 2.054 & 1.876 & 2.071 & 1.482 & 2.071 & 2.071 & 2.429 & 2.464 & 2.065 \\
\hline 3 & 1.197 & 1.804 & 1.143 & $0 . .839$ & 1.214 & 1.286 & 1.928 & 1.606 & 1.377 \\
\hline 4 & 1.733 & 2.054 & 1.571 & 1.017 & 1.571 & 1.500 & 2.214 & 2.321 & 1.748 \\
\hline 5 & 1.983 & 1.804 & 2.000 & 1.840 & 2.286 & 2.358 & 2.643 & 2.107 & 2.127 \\
\hline 6 & 2.519 & 2.054 & 2.429 & 2.018 & 2.643 & 2.572 & 2.929 & 2.822 & 2.498 \\
\hline 7 & 1.661 & 1.983 & 1.500 & 1.375 & 1.786 & 1.786 & 2.429 & 1.964 & 1.810 \\
\hline 8 & 2.197 & 2.233 & 1.929 & 1.553 & 2.143 & 2.000 & 2.714 & 2.678 & 2.181 \\
\hline 9 & 1.911 & 2.161 & 1.572 & 1.483 & 1.429 & 1.500 & 2.000 & 1.607 & 1.708 \\
\hline 10 & 2.447 & 2.411 & 2.000 & 1.661 & 1.786 & 1.714 & 2.286 & 2.321 & 2.078 \\
\hline 11 & 2.125 & 2.590 & 1.500 & 1.196 & 1.286 & 1.143 & 2.071 & 2.178 & 1.761 \\
\hline 12 & 2.125 & 2.590 & 1.500 & 1.196 & 1.286 & 1.143 & 2.071 & 2.178 & 1.761 \\
\hline 13 & 2.375 & 2.340 & 1.929 & 2.018 & 2.000 & 2.001 & 2.500 & 1.964 & 2.141 \\
\hline 14 & 2.911 & 2.590 & 2.358 & 2.197 & 2.358 & 2.215 & 2.786 & 2.679 & 2.511 \\
\hline 15 & 2.054 & 2.518 & 1.429 & 1.554 & 1.501 & 1.429 & 2.286 & 1.821 & 1.824 \\
\hline 16 & 2.590 & 2.768 & 1.858 & 1.732 & 1.858 & 1.643 & 2.572 & 2.536 & 2.194 \\
\hline 17 & 1.339 & 1.804 & 1.500 & 1.553 & 1.428 & 1.571 & 1.428 & 1.392 & 1.502 \\
\hline 18 & 2.661 & 2.054 & 2.786 & 2.732 & 2.857 & 2.857 & 2.429 & 2.607 & 2.623 \\
\hline 19 & 2.482 & 2.661 & 2.358 & 2.411 & 2.143 & 2.429 & 2.143 & 1.821 & 2.306 \\
\hline 20 & 2.232 & 2.911 & 1.929 & 1.589 & 1.429 & 1.571 & 1.714 & 2.035 & 1.926 \\
\hline 21 & 2.089 & 2.197 & 1.358 & 1.875 & 1.572 & 1.286 & 2.143 & 1.750 & 1.784 \\
\hline 22 & 2.482 & 2.090 & 1.929 & 1.982 & 1.857 & 1.571 & 2.143 & 2.250 & 2.038 \\
\hline
\end{tabular}


Table 9. Prediction of Experiments Results for Quality Response: Texture

\begin{tabular}{|c|c|c|c|c|c|c|c|c|c|}
\hline \multirow{3}{*}{ TRIAL } & \multicolumn{8}{|c|}{ UNCONTROLLABLE FACTOR } & \multirow{3}{*}{$\begin{array}{c}\text { RESULT } \\
\text { (AVERAGE) }\end{array}$} \\
\hline & \multicolumn{4}{|c|}{ REPLICATIONS } & \multicolumn{4}{|c|}{ REPLICATIONS } & \\
\hline & 1 & 2 & 3 & 4 & 1 & 2 & 3 & 4 & \\
\hline 1 & 1.018 & 1.179 & 0.768 & 1.090 & 1.179 & 1.125 & 1.179 & 1.768 & 1.163 \\
\hline 2 & 2.054 & 1.393 & 1.233 & 1.626 & 1.964 & 1.590 & 1.679 & 2.232 & 1.721 \\
\hline 3 & 1.054 & 1.464 & 0.590 & 1.340 & 1.393 & 1.661 & 1.393 & 1.804 & 1.337 \\
\hline 4 & 2.090 & 1.678 & 1.054 & 1.876 & 2.179 & 2.125 & 1.893 & 2.268 & 1.895 \\
\hline 5 & 1.339 & 1.322 & 1.375 & 1.697 & 1.678 & 1.304 & 1.679 & 1.875 & 1.533 \\
\hline 6 & 2.375 & 1.536 & 1.840 & 2.233 & 2.464 & 1.768 & 2.179 & 2.339 & 2.092 \\
\hline 7 & 1.375 & 1.607 & 1.197 & 1.947 & 1.893 & 1.839 & 1.893 & 1.911 & 1.708 \\
\hline 8 & 2.411 & 1.821 & 1.661 & 2.483 & 2.678 & 2.304 & 2.393 & 2.375 & 2.266 \\
\hline 9 & 1.339 & 2.533 & 1.304 & 1.554 & 1.036 & 1.661 & 1.607 & 2.375 & 1.676 \\
\hline 10 & 2.375 & 2.747 & 1.768 & 2.090 & 1.822 & 2.125 & 2.107 & 2.839 & 2.234 \\
\hline 11 & 2.411 & 3.032 & 1.590 & 2.340 & 2.036 & 2.661 & 2.322 & 2.875 & 2.408 \\
\hline 12 & 2.411 & 3.032 & 1.590 & 2.340 & 2.036 & 2.661 & 2.322 & 2.875 & 2.408 \\
\hline 13 & 1.660 & 2.676 & 1.911 & 2.161 & 1.536 & 1.839 & 2.107 & 2.482 & 2.046 \\
\hline 14 & 2.697 & 2.890 & 2.375 & 2.697 & 2.321 & 2.304 & 2.607 & 2.946 & 2.605 \\
\hline 15 & 1.696 & 2.961 & 1.732 & 2.411 & 1.750 & 2.375 & 2.322 & 2.518 & 2.221 \\
\hline 16 & 2.732 & 3.175 & 2.197 & 2.947 & 2.536 & 2.840 & 2.822 & 2.982 & 2.779 \\
\hline 17 & 1.125 & 1.113 & 1.375 & 1.768 & 1.678 & 1.446 & 1.107 & 1.375 & 1.373 \\
\hline 18 & 2.447 & 1.185 & 2.625 & 2.661 & 2.750 & 1.554 & 1.893 & 1.911 & 2.128 \\
\hline 19 & 1.696 & 2.393 & 2.232 & 2.197 & 1.535 & 1.732 & 1.964 & 2.089 & 1.980 \\
\hline 20 & 2.447 & 2.750 & 1.911 & 2.376 & 2.036 & 2.554 & 2.179 & 2.482 & 2.342 \\
\hline 21 & 1.732 & 2.893 & 2.196 & 2.804 & 2.036 & 2.268 & 2.179 & 2.482 & 2.324 \\
\hline 22 & 2.411 & 2.678 & 2.232 & 2.483 & 2.107 & 2.018 & 1.964 & 2.803 & 2.337 \\
\hline
\end{tabular}

Based on the average value in Table 9 above, the best level factors combination for flavor and texture is A1 B2 C2 D2 E2 F1 and A2 B1 C1 D2 E2 F2 respectively. Since the best level factors combination for both of quality response is different, then the optimum level factors combination for both of quality response would be determined based on Multi Responses Signal to Noise (MRSN) analysis. Following is the procedure for the MRSN analysis.

Step 1: Calculate lost function $\left(L_{i j}\right)$ because of the change of level factor combination using the following formula: 


$$
L_{i j}=k \frac{1}{n_{i}} \sum_{k=1}^{n_{i}} \frac{1}{y^{2} i j k}
$$

Where $k$ is the ratio of the lost with the quality level improvement.

The quality improvement level for flavor is 0.458 and the texture is 0.440 . With significant cost is IDR 2,000.00 and IDR 1,100.00, then the $k$ value for flavor and texture is 9.535 and 5.682 respectively.

Step 2:Normalise quality loss $\left(C_{i j)}\right.$ of the experiments using the following formula.

$$
\begin{gathered}
C_{i j}=\frac{L_{i j}}{L_{i}^{*}} \\
L_{i}^{*}=\max \left\{L_{i 1}, L_{i 2}, \ldots, L_{i j}\right\}
\end{gathered}
$$

Step 3:Calculate total normalized quality loss (TNQL) of the experiments using the following formula.

$$
T N Q L_{i}=\sum_{i=1}^{m} w_{i} C_{i j}
$$

Where $w_{i}$ is the normalised weight for the quality response $i$.

In this study, normalized weight for flavor and texture was determined through brainstorming among the expert evaluators and has been determined 0.44 and 0.56 respectively.

Step 4:Calculate MRSN of the experiments using the following formula.

$$
M R S N_{j}=-10 \log \left(T N Q L_{j}\right)
$$

Based on the MRSN formula, the optimum level factors combination for the yogurt production process is A2 B1 C1 D2 E2 F1, with the MRSN value is 6.7427. It means that the optimum heating temperature is $95^{\circ} \mathrm{C}$, the heating duration is 20 minutes, the number of yogurt seeds is $7.5 \mathrm{ml}$, incubation temperature is $45^{\circ} \mathrm{C}$, incubation duration is 6 hours, and the number of sugars is 12.5 gram. That factor combination improves the flavor quality level at $16.24 \%$ and the texture quality level at $11.37 \%$.

\section{Conclusion}

From the experiments, it could be concluded that the quality of the yogurt product could be successfully designed by determining optimum level factors combination for the yogurt production process. One of the challenges in multi quality responses is to determine an optimal solution when the level factors combination for every quality response are different. In this study, that challenge could be answered by using the lost function analysis. It means that the proposed solution could be implemented with minimum total additional cost compared to its impact on the undesired effect (noise).

Suggestions for further study related to this study are a determination of the weight for every quality response properly and sensitivity analysis of the change of the weight for every quality response. The sensitivity analysis would help the decision-makers to decide when they have to revise the optimum level factors combination if there is a change in the weight of quality responses. 


\section{References}

Anuyahong, T., Chusak, C., \& Adisakwattana, S. (2020). Incorporation of anthocyanin-rich riceberry rice in yogurts: Effect on physicochemical properties, antioxidant activity and in vitro gastrointestinal digestion. LWT - Food Science and Technology , 1-10.

Behera, A., Sahu, P. S., \& Patel, S. K. (2020). Application of Taguchi methodology for optimization of process parameters in laser bending of Al sheet. Materials Today: Proceedings , 23232327.

Belavendram, N. (1995). Quality By Design: Taguchi Techniques for Industrial Experimention. New Jersey: Prentice-Hall International.

Fakkir Mohamed, M., \& Lenin, K. (2020). Optimization of Wire EDM process parameters using Taguchi technique. Materials Today: Proceedings , 527-530.

Ikeagwuani, C. C., Nwonu, D. C., Ugwu, C. K., \& Agu, C. C. (2020). Process parameters optimization for eco-friendly high strength sandcrete. Heliyon, 1-14.

Khare, S. K., Phull, G. S., \& Agarwal, S. (2020). Optimization the Machining Parameters of Surface Roughness During Micro- EDM by Taguchi Method. Materials Today: Proceedings , 475-479.

Lesme, H., Rannou, C., Famelart, M.-H., Bouhallab, S., \& Prost, C. (2020). Yogurts enriched with milk proteins: Texture properties, aroma release and sensory perception. Trends in Food Science \& Technology, 140-149.

Lordan, R., Vidal, N. P., Pham, T. H., Tsoupras, A., Thomas, R. H., \& Zabetakis, I. (2020). Yoghurt fermentation alters the composition and antiplatelet properties of milk polar lipids. Food Chemistry, 1-12.

Ramesh, K., Singh, S. P., Gnanasekaran, K., \& Kumar, A. S. (2020). Optimization of turning process parameters in machining of heat treated ductile iron bar using Taguchi technique. Materials Today: Proceedings , 2316-2323.

Shunmugasundaram, M., Praveen Kumar, A., Ponraj Sankar, L., \& Sivasankar, S. (2020). Optimization of process parameters of friction stir welded dissimilar. Materials Today: Proceedings , 871-876.

Tribs, A. A., Falcade, L. T., Carvalho, N. S., de Castro Leite Junior, B. R., \& de Oliveira, M. M. (2020). Using stirring and homogenization to improve the fermentation profile and physicochemical characteristics of set yogurt from fresh, refrigerated and frozen/thawed sheep milk. LWT - Food Science and Technology , 1-9.

Wang, X., Kristo, E., \& LaPointe, G. (2020). Adding apple pomace as a functional ingredient in stirred-type yogurt and yogurt drinks. Food Hydrocolloids, 1-10. 